ASTHMA

\title{
Linkage/association study of a locus modulating total serum $\lg$ E on chromosome 14q13-24 in families with asthma
}

\author{
A H Mansur, D T Bishop, S T Holgate, A F Markham, J F J Morrison
}

Thorax 2004;59:876-882. doi: 10.1136/thx.2003.014092

See end of article for authors' affiliations

Correspondence to:

Dr A H Mansur,

Respiratory Department,

Birmingham Heartlands

Hospital, Bordesley Green

East, Birmingham B9 5SS,

UK; adel.mansur@

heartsol.wmids.nhs.uk

Received 11 August 2003

Accepted 24 June 2004

\begin{abstract}
Background: A study was undertaken to validate a locus modulating total serum lgE levels on 14q13-24. Methods: A linkage and association study was performed between total serum lgE and a panel of seven microsatellites which map to the 14q13-24 region in 69 families with asthma recruited from Leeds, UK. Results: Non-parametric, multipoint, sib pair analysis showed no evidence of genetic linkage between the quantitative trait "log lgE" and any of the tested markers. However, a significant association was observed between locus D14S63 (14q23) and total serum IgE ( $p=0.017)$. Allelic analysis showed an association between low total $\operatorname{lgE}$ and allele 157 of D14S63 ( $p=0.01, \mathrm{OR}=0.63,95 \% \mathrm{Cl} 0.44$ to 0.90$)$. Modelling of allele 157 genotypes as a continuous covariate indicated evidence of a significant inverse linear trend across the three genotypes where 157 homozygotes had the lowest mean $\log \lg E(p=0.045)$. Association of D14S63 with log lgE was confirmed in the analysis of a combined dataset of 53 families from Southampton, UK and the 69 families from Leeds (total 122 families). An association was observed at the locus level $(p=0.022)$ and the allelic level where allele 165 showed an association with high total lgE $(p=0.001, O R=3.79,95 \% \mathrm{Cl} 1.54$ to 9.7$)$ and allele 157 showed an association with low total $\lg \mathrm{E}$ $(p=0.041, O R=0.77,95 \% \mathrm{Cl} 0.6$ to 0.99$)$. The transmission disequilibrium test was positive for allele $165(p<0.05)$ and negative for allele $157(p>0.05)$.

Conclusions: Despite the lack of linkage, the findings of this study support the previous observation of a gene(s) at $14 q 23$ that modulates total serum $\lg \mathrm{E}$.
\end{abstract}

A topy is the most common risk factor for asthma and is characterised by the tendency to generate immunoglobulin E (IgE) against environmental allergens. ${ }^{1}$ The close association between total IgE, atopy, and asthma has focused study of the genetics of atopy and asthma on isolating loci controlling IgE expression. ${ }^{2-4} \mathrm{IgE}$ is essential for the development of the inflammatory process that produces symptoms of asthma, allergic rhinitis, and other allergic respiratory disorders. ${ }^{5}{ }^{6}$ Blocking the effect of IgE by anti-IgE monoclonal antibody (omalizumab) is effective in treating moderate to severe asthma and related disorders. ${ }^{7}$ In the population, total serum IgE level distribution is skewed ${ }^{8}$ and modulated by important environmental and genetic factors. ${ }^{9}$ Twin and family based studies have established an important genetic component regulating total serum IgE with an estimated heritability of $0.37-0.84 .{ }^{10}$ The mode of inheritance of total IgE is not known and recessive, ${ }^{11}$ dominant, ${ }^{12}$ codominant, ${ }^{13}{ }^{14}$ and polygenic ${ }^{15}$ inheritance modes have all been postulated. However, the genetic control of IgE is likely to be polygenic, although certain genes might be more important in certain ethnic groups.

There have been numerous reports of linkage or association of the total serum IgE trait to various regions across the genome. These include chromosomes $4 q^{16}{ }^{17} 5 q^{18-20} 6 p^{20}$ $7 q^{2021} 11 q^{2622} 12 q^{20}{ }^{2324} 13 q^{16} 14 q,,^{25} 2616 q,{ }^{1621}$ and Xq. ${ }^{27}$ The extent of the risk ratio for the phenotype at each reported candidate region remains unknown. It has also proved difficult to replicate some of these linkages in different populations. This may reflect the extent of the genetic heterogeneity that exists between different ethnic populations as well as the complexity of the genetic regulation of total serum IgE. Differences in terms of methodology and statistical power are other likely causes of conflict between previous reports.

We have previously reported evidence to suggest linkage between total serum IgE and markers which map to the 14q13-24 region in the middle of 14q. ${ }^{26}$ Markers D14S75 and D14S63 showed suggestive linkage to $\log \operatorname{IgE}(\mathrm{p}=0.034$ and 0.0029 , respectively). However, allelic association was observed with D14S63 allele $165(\mathrm{p}=0.0029)$ on $14 \mathrm{q} 23$ but not D14S75. The evidence for this linkage/association is supported by a recent report of a genome screen showing highly significant linkage between asthma and $14 \mathrm{q} 24 .^{28}$ The locus peak in the latter study is centred on markers D14S588 and D14S603 at 14q24 (LOD score of 4) with a broad base extending between markers D14S1069 and D14S289, centromeric and telomeric respectively (about $4 \mathrm{cM}$ ). This segment is about $4 \mathrm{cM}$ telomeric to marker D14S63 (investigated in the current study). This region contains several genes that encode functions relating to the allergic diathesis, including the nuclear factor kappa B inhibitor (NFKBI), ${ }^{29}$ the transcription factor $\mathrm{FOS}^{30}$ eosinophilic cationic protein $(\mathrm{ECP}),{ }^{31}$ transforming growth factor $\beta_{3}\left(\mathrm{TGF}-\beta_{3}\right),{ }^{32}$ and $a$ disintegrin metalloproteinase (ADAM) domain-20 and -21 gene family which has gained particular importance since the recent report of linkage of ADAM33 to asthma. ${ }^{33}$

To validate our previous finding, we report the results of a further linkage and association study between a panel of microsatellites which map to the $14 \mathrm{q} 13-24$ region and total serum IgE in a separate population of 69 families with asthma from Leeds, UK. We also conducted analyses on a combined dataset of families from Southampton (described in our previous study) and the families from Leeds (presented in this study).

\section{METHODS}

\section{Subjects}

Four extended and 65 nuclear families comprising 139 sib pairs (320 subjects, 175 males) were identified from general practice, hospital outpatient clinics, asthmatic swimming clubs, and media announcements in the West Yorkshire region of Northern England. Families were recruited on the 
basis of the presence of at least two affected sib pairs with doctor diagnosed asthma or the presence of any two (or more) asthmatic members in the family. The four extended families were derived from three generations. All the recruited families were of white ethnic group except for one nuclear family of five members in which the father was of Asian origin and the mother was white. Fathers were not available for clinical assessment in eight families due to divorce or employment circumstances. One father had married twice and had two children from his first marriage and three from his second marriage. The mother from the first marriage was not available for the study. In the rest of the families another five siblings had moved away from home and did not attend the clinical assessment. In the remaining set, 304 individuals provided blood samples for the DNA analysis, 295 for total serum IgE measurement, and skin prick testing was performed on 304 individuals.

Only children over the age of 5 years were considered suitable for the study because younger children are more difficult to characterise clinically. The mean age of all subjects was 29.2 years (range 5-84). All families contained more than one asthmatic member. Those with doctor diagnosed asthma constituted $59.1 \%$ of the studied population.

A full, verbal and written explanation of the study was given to each family member. The study was approved by the ethical committee at St James's University Hospital. The children gave informed verbal consent and the parents gave informed written consent.

In the second stage of the study we combined the Leeds families with 53 families with asthma recruited from Southampton. The Southampton families included a total of 320 individuals (155 males) and have been previously described elsewhere. ${ }^{26}$

\section{Clinical parameters of Leeds families}

Each family member completed a structured written questionnaire (MRC Respiratory Health) on atopic and asthmatic symptoms. Total serum IgE was measured in 295 subjects by the radioimmunoassay method (Pharmacia CAP System IgE RIA). Because of the wide range of the participants' ages, log total IgE values for all subjects were age/sex adjusted by linear standardisation to male subjects at 20 years of age. For the purpose of association analysis, total IgE was also analysed as a dichotomy using the age dependent 70th percentile as a cut off level. ${ }^{34}$ In this qualitative trait the age dependent normal range values for total serum IgE (in kU/l) used were as follows: less than 50 for $<6$ years; less than 100 for 6-16 years; and less than 125 for over 16 years.

The atopic status was determined by measuring skin reactivity to common allergens. Skin prick testing by stylet was performed for 14 common allergens (Dermatophagoides pteronyssinus, Dermatophagoides farinae, mixed grass, mixed trees, Alternaria, Cladosporium, horse, feathers, egg white, egg yolk, cow's milk, Aspergillus fumigatus, cat fur and dog fur; Bayer Corporation). The major and minor axes of each wheal were recorded. Short acting antihistamines were discontinued for 2 days before the test and long acting antihistamines were discontinued as specified. Atopy was defined as a positive skin reaction ( $3 \mathrm{~mm}$ or more than negative saline control) to one or more of the 14 tested antigens regardless of total IgE values.

\section{Molecular methods and genotypic data}

DNA extraction was performed from peripheral blood leucocytes collected in EDTA-containing tubes using the phenol-chloroform method. DNA was available from 304 individuals who were genotyped for the polymorphic microsatellites D14S49, D14S75, D14S978, D14S276, D14S750, D14S63, and D14S251 which span the 14q13-24 region. The

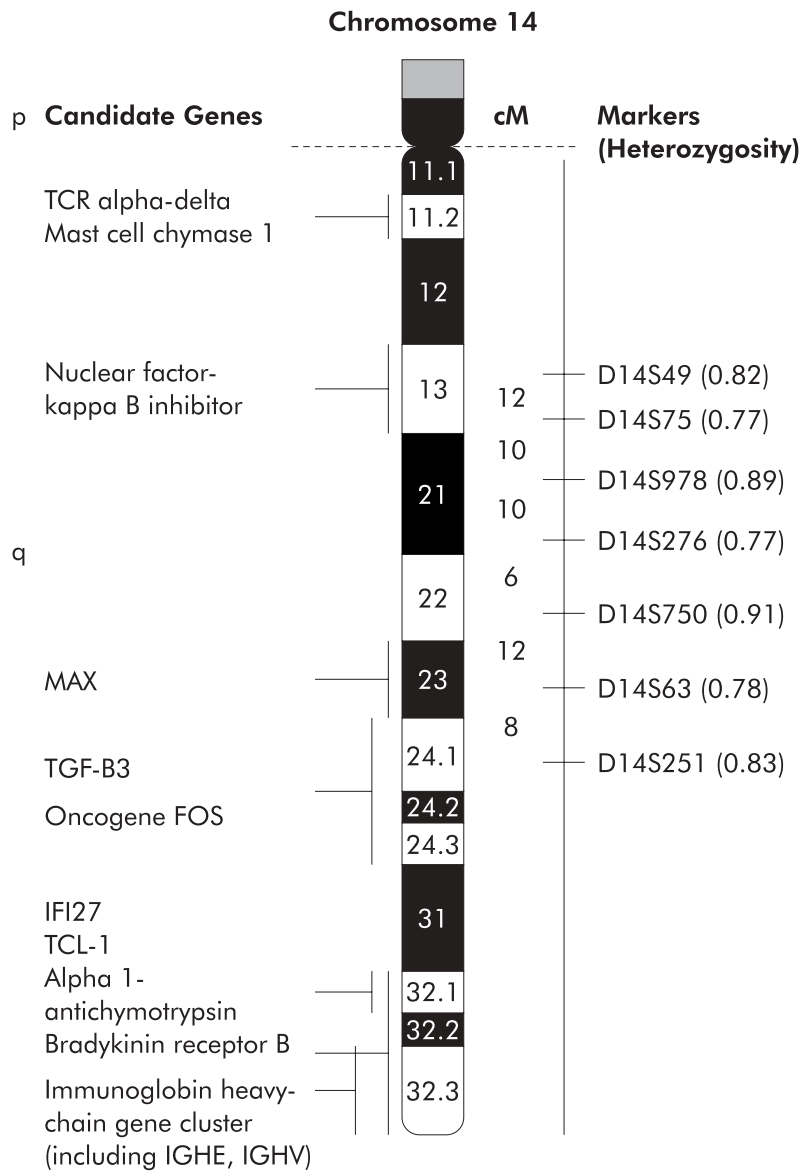

Figure 1 Chromosome 14q map showing the cytogenetic regions, the corresponding relative positions and order of the markers used in this study, and the candidate genes mapped to this chromosome. The (sex averaged) distance in centi-Morgans (CM) and the order of the markers correspond to published maps. ${ }^{44}{ }^{46}$ The relative positions of the

candidate genes are based on data obtained from the Genetic Location Data Base (LDB). ${ }^{46} \mathrm{MAX}$, oncogene myc-associated protein; TGF- $\beta_{3}$, transforming growth factor- $\beta_{3} ; \mathrm{IFI} 27$, interferon-alpha inducible factor 27; TCL-1, T cell lymphoma factor 1; IGHE, immunoglobulin heavy chain epsilon locus; IGHV, immunoglobulin heavy chain variable locus.

genetic distance between these markers is shown in fig 1 . Microsatellite primer sequences, their polymerase chain reaction (PCR) amplification conditions, and genetic location data were retrieved from the Human Genome Data Base (GDB). Primers were purchased from Oswel (University of Southampton, UK). PCR amplification of DNA conditions are as previously described.$^{26}$ To confirm accuracy of typing and rule out variation in allele sizing between gels, a control DNA was incorporated in all runs. Data generated by Genotyper software were subsequently analysed by the Genetic Analysis System (GAS) program. Inconsistency in allele designation within families was checked by the program and ambiguities were resolved by further genotyping. Haplotypes were also constructed for all families studied. Two point LOD scores between the markers and marker order were determined using the Vitesse Engine Routine of GAS which provided similar results to published maps of $14 \mathrm{q} .{ }^{35}$

\section{Statistical methods}

In the first stage of the study, linkage and association between the seven microsatellite markers and total serum IgE were tested in the Leeds families. Linkage analysis for the quantitative trait, $\log$ IgE, was conducted using multipoint quantitative sib pair analysis. Association analysis was 
Table 1 Correlation matrix for log total serum lgE and lgE dependent phenotypes in the Leeds families

\begin{tabular}{|c|c|c|c|c|c|c|c|c|}
\hline & Asthma & Atopy & $\lg E$ & $D f$ & $D p$ & Grass & Cat & Alternaria \\
\hline Asthma & 1.00 & 0.43 & 0.43 & 0.47 & 0.44 & 0.30 & 0.38 & 0.16 \\
\hline Atopy & & 1.00 & 0.58 & 0.63 & 0.71 & 0.81 & 0.62 & 0.41 \\
\hline $\lg \mathrm{E}$ & & & 1.00 & 0.48 & 0.53 & 0.50 & 0.50 & 0.27 \\
\hline$D f$ & & & & 1.00 & 0.86 & 0.50 & 0.60 & 0.26 \\
\hline$D p$ & & & & & 1.00 & 0.55 & 0.68 & 0.28 \\
\hline Grass & & & & & & 1.00 & 0.56 & 0.48 \\
\hline Cat & & & & & & & 1.00 & 0.41 \\
\hline Alternaria & & & & & & & & 1.00 \\
\hline
\end{tabular}

Df, Dermatophagoides farinae; Dp, Dermatophagoides pteronyssinus.

Associations between "doctor diagnosed" asthma, $\log \lg E$ quantitative trait, and the qualitative traits of atopy or skin prick test reactivity to $D$ farinae, $D$

pteronyssinus, grass, cat, and Alternaria alternata were examined using covariate analysis and a correlation matrix. The results showed close correlation between

$\log \lg E$ and other dependent traits in which all Pearson's $r$ values were strongly significant $(p<0.001)$.

performed using $\chi^{2}$ tests for the qualitative trait, total serum IgE. Further association analysis between total serum IgE and Dl4S63 was conducted on a combined dataset comprising families from Leeds and Southampton.

\section{Multipoint quantitative sib pair analysis}

Data were analysed using the GAS package version 2.0 (Alan Young, University of Oxford). For the quantitative locus IgE, in the absence of a specified genetic model, non-parametric linkage analysis was performed using the Elston-Haseman algorithm. ${ }^{36}$ In this method siblings sharing marker alleles near the quantitative locus are more likely to have similar quantitative values than the non-sharing siblings. The mean value of the difference between siblings should decrease as the fraction of alleles shared increases. A slope is generated by a least squares fit using allele sharing as the independent variable and trait difference as the dependent variable. A significant slope would indicate linkage. Data analysis was performed using the SIBIHE routine of GAS. This routine combines the Elston-Haseman algorithm with sib pair interval mapping and has been described elsewhere. ${ }^{26}$

\section{Association analysis}

Association analysis was performed using the SPSS (7.0) statistical package (SPSS 7.01995 for VAX/VMS, SPSS Inc, USA). Data were initially analysed at the locus level. Significantly associated loci were subsequently subjected to allelic association tests to identify the associated allele. The Monte Carlo simulation test was conducted to evaluate the significance of positive results obtained using multi-allelic markers.

The distribution of alleles at each microsatellite locus in the affected (total IgE over 70th percentile) versus unaffected individuals (total IgE under 70th percentile) was examined by performing combined $\chi^{2}$ tests on the allelic classes of each marker. To reduce the number of comparisons performed, rare alleles with a frequency of less than 5\% for each marker were pooled and included in the analysis as a single class labelled "rare alleles". This analytical approach resulted in reduction of the overall number of comparisons, since only one statistical test per locus was required to test for association. An approximation for Bonferroni correction using seven comparisons (that is, the number of markers tested) requires an empirical level of $p=0.007$ to declare significant association with any marker. However, for loci D14S75 and D14S63 we considered $\mathrm{p}=0.05$ to be significant since these two markers constitute a prior hypothesis. ${ }^{26}$

The dichotomised IgE definition was further analysed to define the associated allele(s) and genotype(s) within loci which showed evidence of association at the $p=0.05$ level using $\chi^{2}$ tests. The Monte Carlo simulation test (using CLUMP program) was performed to evaluate the significance of the $\chi^{2}$ values obtained with this allelic association analysis (to correct for multiple testing resulting from using multiallelic loci). ${ }^{37}$

We also conducted allelic association analysis for the quantitative locus $\log$ IgE using the Mann-Whitney U test. The transmission disequilibrium test (TDT) was also performed for marker D14S63. ${ }^{38}$ In the TDT a parent heterozygous for an associated allele $\mathrm{Al}$ and non-associated allele A2 should more often transmit Al than A2 to an affected child-that is, expressing $>70$ th percentile total IgE. In this analysis all affected children were treated as independent observations, summing their transmitted and non-transmitted allele, and significance calculated using an exact two sided binomial distribution. The QTDT program was also used to test for TDT between the quantitative $\log$ IgE and marker D14S63 using the linear orthogonal association model..$^{39}$ This program is also used here to test for potential population stratification which may arise due to familial correlation.

\section{RESULTS}

\section{Clinical assessment of Leeds families}

Data analysis was based on the 69 families (total 320 members, 175 males). Clinical data were obtained from 304 participants and DNA genotyping was successful in 295. Total serum IgE distribution was skewed with levels ranging from $<2$ to $>2000 \mathrm{kU} / \mathrm{l}$ (mean (SD) 409.3 (579.9)). Nineteen subjects had a total IgE of $>2000 \mathrm{kU} / \mathrm{l}$ ( 16 children and three parents) while seven subjects had a total IgE of $<2 \mathrm{kU} / \mathrm{l}$ (three children and four parents). In these cases the IgE level was assumed to be $2000 \mathrm{kU} / \mathrm{l}$ or $2 \mathrm{kU} / \mathrm{l}$, respectively. All but one of the subjects with $>2000$ IgE were asthmatic $(94.7 \%)$ compared with an overall average of asthma prevalence in the Leeds families of $59.1 \%$. They also had an increased mean number of wheeze episodes in the preceding 12 months (7.6 per year) and $57.9 \%$ suffered previous severe attacks that required hospital admission. The prevalence of eczema and rhinitis were also markedly increased $(73.7 \%$ and $80 \%$, respectively).

In the Leeds families there was a higher proportion of asthmatics among children than in the other age groups and there was close correlation between asthma, atopy, $\log \operatorname{IgE}$, and specific skin reactivity to common allergens (table 1). Using "doctor diagnosed asthma" as the dependent variable, we conducted forward multiple logistic regression analysis in a model that included age, sex, log(e) IgE (age uncorrected), and the traits $D$ pteronyssinus, $D$ farinae, mixed grass and cat positive skin test reactivity as well as atopy. The best fit model for asthma was that including $\log (e) \operatorname{IgE}(p=0.002)$, $D$ farinae $(\mathrm{p}=0.000)$, and age $(\mathrm{p}=0.000)$.

\section{Multipoint sib pair analysis in Leeds families}

Table 2 shows the results of the multipoint sib pair analysis for the seven loci and provides evidence against linkage between any of these loci and log IgE. These results remained 
Table 2 Multipoint sib pair analysis for the quantitative trait $\log \lg E$ (Haseman-Elston method)

\begin{tabular}{llllll}
\hline Location $(\boldsymbol{\theta})$ & Marker & M & C & Intercept & p value \\
\hline 0.00 & D14S49 & 0.10 & 0.81 & -7.9 & 0.71 \\
0.12 & D14S75 & -0.03 & 0.95 & 36 & 0.45 \\
0.20 & D14S978 & -0.12 & 1.00 & 8.4 & 0.26 \\
0.26 & D14S276 & -0.04 & 0.96 & 24 & 0.42 \\
0.29 & D14S750 & -0.06 & 0.97 & 17 & 0.39 \\
0.34 & D14S63 & 0.10 & 0.83 & -8.1 & 0.7 \\
0.36 & D14S251 & -0.02 & 0.94 & 41 & 0.45 \\
\hline
\end{tabular}

The analysis is based on 107 sib pairs (mean (SD) age 16.75 (8.84) years, range 6.0-52). The genetic distance by recombination fraction between the markers was estimated from the genotype data in these families using the Vitesse Engine Routine of GAS. Regression by the least square fitting formula: $D=M^{*} S h+C$ where $D$ is the difference in $\log \lg E$ values of siblings, $S h$ is the alleles shared. $M<0=>$ increased sharing tend to reduce $D$. Intercept is value Sh satisfying $0=M^{*} S h+C$. The results show evidence against linkage between the analysed markers and log $\lg \mathrm{E}$.

unchanged even when families with incomplete parental genotype data were excluded from the analysis (data not shown).

\section{Locus association analysis in Leeds families}

Table 3 shows the results of association analyses at the locus level. None of the markers analysed showed association at the $\mathrm{p}=0.007$ level (Bonferroni correction for seven tests). However, marker D14S63 showed significant association with the qualitative trait "total IgE" at a level of $\mathrm{p}=0.017$.

\section{Allelic association analysis in Leeds families}

Association analysis between D14S63 alleles and total IgE shows significant association between allele 157 and total IgE (odds ratio (OR) 0.63 (95\% CI 0.44 to 0.9 ), $\mathrm{p}=0.01$ ). Plotting allele 157 genotypes against $\log$ IgE revealed a linear inverse relationship in which allele 157 homozygotes had the lowest level of mean $\log \operatorname{IgE}(p=0.045$, fig 2$)$.

\section{Association analysis of D14S63 in the combined dataset}

The D14S63 microsatellite has 11 alleles and a heterozygosity index of 0.79. In the Leeds and Southampton families we observed 10 common and rare alleles. The rare allele 153 was not observed in these families. D14S63 allele frequency in the

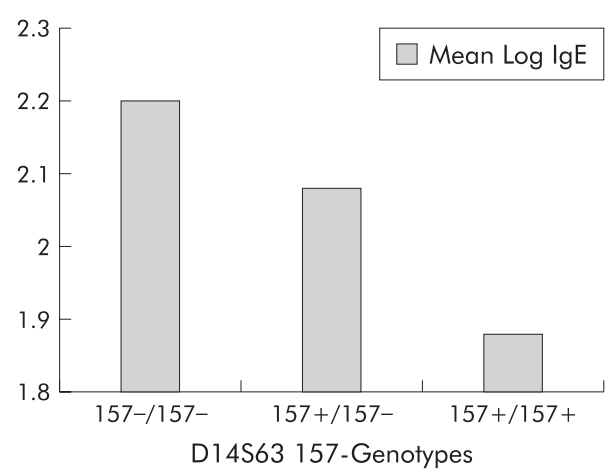

Figure 2 Mean $\log \lg E$ across the D14S63 allele 157 genotypes. An inverse proportional relationship was seen between the number of allele 157 copies inherited and mean log serum lgE level. The analysis was performed using the non-parametric Kruskal-Wallis test. $\chi^{2}=6.22$, $\mathrm{df}=2, \mathrm{p}=0.045$.

Leeds and Southampton families is consistent with that observed in the CEPH families. ${ }^{40}$

Association analysis between total IgE and Di4S63 was conducted in a dataset comprised of families from Leeds and Southampton (total of 122 families, 532 individuals with

Table 3 Association analysis with the qualitative trait lgE using the $\chi^{2}$ method

\begin{tabular}{|c|c|c|c|c|c|c|c|c|c|c|c|}
\hline Locus (alleles) & & & & & & & & No $\left(\% \lg E_{+}\right)$ & $\chi^{2}$ & $\mathrm{df}$ & p value \\
\hline \multicolumn{12}{|l|}{ D14S49 (13) } \\
\hline Allele & 116 & 118 & 122 & 124 & 128 & 134 & Rare alleles & $279(53 \%)$ & 9.37 & 6 & 0.15 \\
\hline Frequency & $53 / 90(59 \%)$ & $22 / 34(65 \%)$ & $51 / 90(57 \%)$ & $78 / 153(51 \%)$ & $27 / 63(43 \%)$ & $24 / 39(62 \%)$ & $41 / 89(46 \%)$ & & & & \\
\hline \multicolumn{12}{|l|}{ DI4S75 (10) } \\
\hline Allele & 172 & 174 & 180 & 182 & 186 & Rare alleles & & & & & \\
\hline Frequency & $32 / 59(54 \%)$ & $75 / 137(55 \%)$ & $19 / 32(59 \%)$ & $87 / 176(49 \%)$ & $37 / 73(51 \%)$ & $40 / 75$ (53\%) & & $276(53 \%)$ & 1.7 & 5 & 0.89 \\
\hline \multicolumn{12}{|l|}{ D14S978 (11) } \\
\hline Allele & 231 & 248 & 250 & 252 & 254 & 256 & Rare alleles & & & & \\
\hline Frequency & $39 / 69$ (57\%) & $28 / 63(44 \%)$ & $26 / 55(47 \%)$ & $79 / 147(54 \%)$ & $60 / 104(58 \%)$ & $41 / 76(54 \%)$ & $25 / 50(50 \%)$ & $282(53 \%)$ & 4.07 & 6 & 0.67 \\
\hline \multicolumn{12}{|l|}{ D14S978 (11) } \\
\hline Allele & 87 & 89 & 91 & 93 & 95 & Rare alleles & & & & & \\
\hline Frequency & $32 / 44(72 \%)$ & $82 / 159(52 \%)$ & $115 / 221(52 \%)$ & $25 / 52(48 \%)$ & $43 / 80(54 \%)$ & $3 / 10(30 \%)$ & & $283(53 \%)$ & 10.09 & 5 & 0.07 \\
\hline \multicolumn{12}{|l|}{ D14S750 (8) } \\
\hline Allele & 152 & 156 & 160 & 164 & 168 & Rare alleles & & & & & \\
\hline Frequency & $76 / 138(55 \%)$ & $62 / 98(63 \%)$ & $56 / 121(46 \%)$ & $48 / 87(55 \%)$ & $35 / 73(48 \%)$ & $17 / 35(49 \%)$ & & 276 (53\%) & 7.81 & 5 & 0.17 \\
\hline \multicolumn{12}{|l|}{ D14S63 (10) } \\
\hline Allele & 157 & 159 & 161 & 163 & 167 & Rare alleles & & & & & \\
\hline Frequency & $99 / 215(46 \%)$ & $23 / 33(70 \%)$ & $21 / 49(43 \%)$ & $46 / 78(59 \%)$ & $57 / 94(61 \%)$ & $38 / 67(57 \%)$ & & $268(53 \%)$ & 13.73 & 5 & 0.017 \\
\hline \multicolumn{12}{|l|}{ D14S251 (11) } \\
\hline Allele & 300 & 302 & 304 & 310 & Rare alleles & & & & & & \\
\hline Frequency & $97 / 186(52 \%)$ & $81 / 151$ (54\%) & $39 / 63(62 \%)$ & $44 / 87(51 \%)$ & $32 / 86(37 \%)$ & & & $277.5(53 \%)$ & 3.27 & 4 & 0.62 \\
\hline
\end{tabular}


Table 4 Association analysis for D14S63 and IgE in the combined dataset of families from Leeds and Southampton

\begin{tabular}{llllll}
\hline & $\begin{array}{l}\text { Frequency of } \\
\text { lgE+/total }\end{array}$ & $\begin{array}{l}\text { Odds ratio } \\
(95 \% \mathrm{Cl})\end{array}$ & df & $\chi^{2}$ test & p value \\
\hline $\begin{array}{l}\text { Allele } 157+ \\
\text { Allele } 157-\end{array}$ & $\begin{array}{l}206 / 451(45.7 \%) \\
320 / 613(52 \%)\end{array}$ & $0.77(0.6$ to 0.99$)$ & 1 & 4.17 & 0.041 \\
Allele $165+$ & $25 / 32(78 \%)$ & $3.79(1.54$ to 9.7$)$ & 1 & 9.71 & 0.0018 \\
Allele $165-$ & $501 / 1032(49 \%)$ & & & & \\
\hline
\end{tabular}

complete D14S63 genotypes and IgE measurement data). Using $\chi^{2}$ tests for the qualitative trait (total IgE), the D14S63 locus showed an association with total $\operatorname{IgE}(p=0.022)$. Similar results were obtained using the non-parametric Kruskal-Wallis test for the quantitative trait $\log \operatorname{IgE}$ $\left(\chi^{2}=15.3, \mathrm{df}=7, \mathrm{p}=0.033\right)$. Allelic analysis showed a significant association between allele 165 and high mean $\log \operatorname{IgE}(p=0.0018)$ and between allele 157 and low mean $\log \operatorname{IgE}(p=0.041$, table 4$)$.

The transmission disequilibrium test for the qualitative IgE trait was positive for allele 165 (transmitted from a parent to affected child in 16 cases and non-transmitted in six cases $(\mathrm{p}<0.05))$. However, the transmission disequilibrium test for allele 157 was negative. Similarly, the quantitative trait log IgE showed positive TDT for allele 165 but not 157. The QTDT program (Abecasis 1998-2002) was used in the latter analysis to conduct linear orthogonal association test for transmission disequilibrium between D14S63 alleles and log IgE. For allele 165, the null model logarthmic likelihood (LnLk) $=570.77$ $(523 \mathrm{df})$ and the full model LnLk $=566.64(522 \mathrm{df})$, $\chi^{2}=8.25, p=0.0041$, Bonferroni significance level $=0.0281$, but not with allele 157. The mean (SD) $\log \operatorname{IgE}$ in allele $165(+)$ subjects of $2.38(0.61)$ is significantly higher than that of allele $157(+)$ subjects $(1.92(0.75), p=0.001)$.

\section{DISCUSSION}

Using linkage and association approaches we have explored the reproducibility of our previous report of linkage and association of total serum IgE to the $14 q 13-24$ region in an independent set of families with asthma recruited from Leeds. We observed no evidence of linkage with any of the examined markers. However, at the locus level, marker D14S63 alone showed significant association with total IgE. Allelic analysis revealed significant association of D14S63 allele 157 with total IgE. Plotting the latter allele genotypes against $\log$ IgE demonstrated a significant inverse linear relationship. D14S63 locus association with total IgE was also observed in analysis of a combined dataset of families recruited from both Leeds and Southampton. In these families, allele 165 had significant association with high mean IgE while allele 157 had significant association with low mean IgE. The transmission disequilibrium test was positive for allele 165 but not for allele 157. The results of this study are therefore in agreement with our previous finding of a gene(s) modulating total serum IgE at 14q23.

The two sets of families were recruited along similar criteria and are similar in terms of age structure, percentage of asthmatics, and mean total IgE levels. The lack of linkage in the Leeds families is unlikely to be caused by clinical differences from Southampton families. Replication of genetic linkage in complex traits has proved difficult due to disease heterogeneity and methodological difficulties. ${ }^{41}$ Lack of power due to the relatively small number of families examined is a likely cause here, since replication of linkage to a heterogeneous trait would require an emphatically larger number of families than those originally studied. ${ }^{42}$ In



Figure 3 Chromosome 14q23-24 map showing the cytogenetic regions, the corresponding relative positions and order of microsatellites, and some of the local candidate genes. The relative positions of the microsatellites and the candidate genes are based on data obtained from the Genetic Location Data Base (LDB) (http://cedar.genetics.soton.ac.uk/public html/ldb.html) ${ }^{46}$ and the Ensambl Data Base (www.ensamble.org). ${ }^{35}$ The positively linked microsatellites to asthma in the study by Hakonarson et a ${ }^{\overline{P B}}$ are underlined and their relative position to microsatellite DI 4S63 is illustrated. HIF1A, hypoxia inducible factor-1 $\alpha$; RBM8, RNA binding motif protein 8A; ZFP46, zinc finger protein 46; MAX, oncogene myc-associated protein; MNAT1, ménage à trois 1; ECP, eosinophilic cationic protein; ADAM20-21, a disintegrin and metalloproteinase domain 20 and domain 21; GPX2, glutathione peroxidase; HSPA2, heat shock 70 kDa protein 2; BCRP1, breakpoint cluster region protein 1; ARG2, arginase II; TGF- $\beta_{3}$, transforming growth factor $\beta_{3}$. 
contrast, association studies are considered more powerful than linkage studies as they involve direct analysis of polymorphism within a gene of interest or of markers in strong linkage disequilibrium with such a gene. ${ }^{42}$ However, a significant result should be interpreted with caution. Statistical association could result from a causal relationship between an expressed marker allele and the pathogenesis of disease; linkage disequilibrium between the marker locus and an unobserved susceptibility locus; or a false association secondary to population stratification or admixture. In the current studies we used unaffected individuals from asthmatic families as internal controls to overcome any stratification related confounders. In addition, we observed no evidence for "in between" family stratification in either set of families which enhances the validity of the general allelic association approach adopted in this study. The use of multiple, highly polymorphic markers is more informative, but inevitably increases the number of comparisons performed to test for an association. We limited the total number of tests performed by conducting a single test of association for each microsatellite (total of seven tests). Within each locus, rare alleles of $<5 \%$ frequency were combined and analysed as a single trait to reduce the total number of allelic classes.

Only locus Dl4S63 had evidence of association at the empirical significance level of $p<0.05$. We considered this result significant as it constitutes a primary hypothesis. D14S63 is a highly polymorphic microsatellite comprised of 11 alleles with a heterozygosity index of 0.79 . The common allele 157 (frequency $=0.43$ ) showed association with low mean $\log \operatorname{IgE}$ in the Leeds families and the rare allele 165 (frequency $=0.025$ ) showed association with high mean log IgE in the Southampton families. ${ }^{26}$ The mean log IgE level in allele $157(+)$ subjects was concordant in the two sets of families and was significantly lower than that of allele 165(+) subjects. In the total population the mean $\log$ IgE in allele $165(+)$ subjects was 2.38 compared with 1.92 in allele $157(+)$ subjects. The common 157 allele may therefore be in linkage disequilibrium with a wild-type allele resulting in low total serum IgE while the rare 165 allele may be in linkage disequilibrium with a mutant allele that encodes a protein causing high total serum IgE.

In addition to our data, a recent genome screen of 175 extended Icelandic families by Hakonarson et al ${ }^{28}$ using 976 microsatellites has identified chromosome $14 \mathrm{q} 24$ as an asthma locus with an allele sharing LOD score of 4.00. The report of linkage/association by two independent research groups to the same genomic region should be considered as confirmatory for the presence of an asthma susceptibility locus at $14 \mathrm{q} 23-24 .^{43}$ However, although our population is comprised of families with asthma, the linkage/association we observed is with total IgE rather than asthma. Figure 3 illustrates the cytogenetic map of chromosome 14q23-24 and depicts the relative position of microsatellites used in this study and those in the study by Hakonarson et al. ${ }^{28}$ This region contains numerous genes that contribute to the control of IgE and susceptibility to asthma, including the ADAM20-21, ECP, and TGF $\beta_{3}$. Further fine mapping of the region and analysis of these candidate genes is now required to isolate and confirm the culprit gene.

In conclusion, this study presents further evidence for a significant association (but no linkage) between the D14S63 locus and IgE. The findings of this study support our previous observation of a gene(s) at $14 \mathrm{q} 23$ that modulates total serum IgE.

\section{ACKNOWLEDGEMENTS}

This paper is dedicated to the memory of J F J Morrison. The authors thank the families from Leeds and Southampton for their generous participation in this study; David Campbell, Sarah Perry and Melanie Shatrath at the Molecular Medicine Unit, St James's University Hospital, Leeds for assistance with the characterisation of the Leeds families; Grange Williams and Alexander Turner for help with genotyping; Patricia Puzycz and H C Gooi for kindly performing total serum IgE measurement, and the genetics team at Southampton General Hospital led by Newton Morton for providing clinical information and DNA on the Southampton families.

\section{Authors' affiliations}

A H Mansur, Respiratory Department, Birmingham Heartlands Hospital, Birmingham B9 5SS, UK

D T Bishop, ICRF, Genetic Epidemiology Laboratory, St James's University Hospital, Leeds, UK

S T Holgate, Medical Specialities (RCMB), Southampton General Hospital, Southampton, UK

A F Markham, Molecular Medicine Unit, University of Leeds, Clinical Sciences Building, St James's University Hospital, Leeds, UK

J F J Morrison (deceased), AstraZeneca, Macclesfield, Cheshire, UK

Work in the authors' laboratory was supported by the National Asthma Campaign, MRC, Imperial Cancer Research Fund, and Yorkshire Cancer Research.

\section{REFERENCES}

1 Ishizaka K, Ishizaka T. IgE and reaginic hypersensitivity. Ann NY Acad Sci $1971 ; 190: 443-56$.

2 Johansson SG, Bennich HH, Berg T. The clinical significance of IgE. Prog Clin Immunol 1972;1:157-81.

3 Burrows B, Martinez FD, Halonen M, et al. Association of asthma with serum lgE levels and skin-test reactivity to allergens. N Engl J Med 1989;320:271-7.

4 Sears MR, Burrows B, Flannery EM, et al. Relation between airway responsiveness and serum $\lg \mathrm{E}$ in children with asthma and in apparently normal children. N Engl J Med 1991;325:1067-71.

5 Busse WW, Coffman RL, Gelfand EW, et al. Mechanisms of persistent airway inflammation in asthma. A role for T cells and T-cell products. Am J Respir Crit Care Med 1995; 152:388-93.

6 Busse WW. Inflammation in asthma: the cornerstone of the disease and target of therapy. J Allergy Clin Immunol 1998;102:S17-22.

7 Schulman ES. Development of a monoclonal anti-immunoglobulin E antibody (omalizumab) for the treatment of allergic respiratory disorders. Am J Respir Crit Care Med 2002;164:S6-11.

8 Barbee RA, Halonen M, Lebowitz $M$, et al. Distribution of $\lg E$ in a community population sample: correlations with age, sex, and allergen skin test reactivity. $J$ Allergy Clin Immunol 1981;68:106-11.

9 Hagel I, Lynch NR, Di Prisco MC, et al. Ascaris reinfection of slum children: relation with the lgE response. Clin Exp Immunol 1993;94:80-3.

10 Bazaral M, Orgel HA, Hamburger RN. IgE levels in normal infants and mothers and an inheritance hypothesis. J Immunol 1971;107:794-801.

11 Dizier MH, Hill M, James A, et al. Detection of a recessive major gene for high lgE levels acting independently of specific response to allergens. Genet Epidemiol 1995;12:93-105.

12 Gerrard JW, Rao DC, Morton NE. A genetic study of immunoglobulin E. Am J Hum Genet 1978;30:46-58.

13 Meyers DA, Bias WB, Marsh DG. A genetic study of total lgE levels in the Amish. Hum Hered 1982;32:15-23.

14 Martinez FD, Holberg CJ, Halonen M, et al. Evidence for Mendelian inheritance of serum lgE levels in Hispanic and non-Hispanic white families. Am J Hum Genet 1994;55:555-65.

15 Hasstedt SJ, Meyers DA, Marsh DG. Inheritance of immunoglobulin E: genetic model fitting. Am J Med Genet 1983;14:61-6.

16 Daniels SE, Bhattacharrya S, James A, et al. A genome-wide search for quantitative trait loci underlying asthma. Nature 1996;383:247-50.

17 Grimbacher B, Schaffer AA, Holland SM, et al. Genetic linkage of hyper-lgE syndrome to chromosome 4. Am J Hum Genet 1999;65:735-44.

18 Marsh DG, Neely JD, Breazeale DR, et al. Linkage analysis of IL4 and other chromosome $5 \mathrm{q} 31.1$ markers and total serum immunoglobulin $E$ concentrations. Science 1994;264:1152-6.

19 Meyers DA, Postma DS, Panhuysen $\mathrm{Cl}$, et al. Evidence for a locus regulating total serum IgE levels mapping to chromosome 5. Genomics 1994;23:464-70.

20 Xu J, Postma DS, Howard TD, et al. Major genes regulating total serum immunoglobulin E levels in families with asthma. Am J Hum Genet 2002;67: $1163-73$

21 Ober C, Cox NJ, Abney M, et al. Genome-wide search for asthma susceptibility loci in a founder population. The Collaborative Study on the Genetics of Asthma. Hum Mol Genet 1998;7:1393-8.

22 Young RP, Sharp PA, Lynch JR, et al. Confirmation of genetic linkage between atopic lgE responses and chromosome 11 q13. J Med Genet 1992;29:236-8.

23 Barnes KC, Neely JD, Duffy DL, et al. Linkage of asthma and total serum IgE concentration to markers on chromosome 12q: evidence from Afro-Caribbean and Caucasian populations. Genomics 1996;37:41-50

24 Wilkinson J, Grimley S, Collins A, et al. Linkage of asthma to markers on chromosome 12 in a sample of 240 families using quantitative phenotype scores. Genomics 1998;53:251-9. 
25 Moffatt MF, Hill MR, Cornelis F, et al. Genetic linkage of T-cell receptor alpha/ delta complex to specific lgE responses. Lancet 1994;343:1597-600.

26 Mansur AH, Bishop DT, Markham AF, et al. Suggestive evidence for genetic linkage between lgE phenotypes and chromosome 14q markers. Am J Respir Crit Care Med 1999;159:1796-802.

27 Wist M, Fischer G, Immervoll T, et al. A genome-wide search for linkage to asthma. German Asthma Genetics Group. Genomics 1999;58:1-8.

28 Hakonarson H, Bjornsdottir US, Halapi E, et al. A major susceptibility gene for asthma maps to chromosome 14q24. Am J Hum Genet 2003;71:483-91.

29 Le Beau MM, lto $C$, Cogswell $P$, et al. Chromosomal localization of the genes encoding the p50/p105 subunits of NF-kappa B (NFKB2) and the I kappa B/ MAD-3 (NFKBI) inhibitor of NF-kappa B to $4 q 24$ and 14q13, respectively. Genomics 1992; 14:529-31.

30 Demoly P, Basset-Seguin N, Chanez P, et al. c-fos proto-oncogene expression in bronchial biopsies of asthmatics. Am J Respir Cell Mol Biol 1992;7:128-33.

31 Mastrianni DM, Eddy RL, Rosenberg HF, et al. Localization of the human eosinophil Charcot-Leyden crystal protein (lysophospholipase) gene (CLC) to chromosome 19 and the human ribonuclease 2 (eosinophil-derived neurotoxin) and ribonuclease 3 (eosinophil cationic protein) genes (RNS2 and RNS3) to chromosome 14. Genomics 1992;13:240-2.

32 Wu CY, Brinkmann V, Cox D, et al. Modulation of human lgE synthesis by transforming growth factor-beta. Clin Immunol Immunopathol 1992;62:277-84.

33 Van Eerdewegh P, Little RD, Dupuis J, et al. Association of the ADAM33 gene with asthma and bronchial hyperresponsiveness. Nature 2003:418:426-30
34 Zetterstrom O Johansson SG. IgE concentrations measured by PRIST in serum of healthy adults and in patients with respiratory allergy. A diagnostic approach. Allergy 1981;36:537-47.

35 Clamp M. Andrews D, Barker D, et al. Ensembl 2002: accommodating comparative genomics. Nucleic Acids Res 2003;31:38-42.

36 Haseman JK, Elston RC. The investigation of linkage between a quantitative trait and a marker locus. Behav Genet 1972;2:3-19.

37 Sham PC, Curtis D. An extended transmission/disequilibrium test (TDT) for multi-allele marker loci. Ann Hum Genet 1995:59:323-36.

38 Spielman RS, McGinnis RE, Ewens WJ. Transmission test for linkage disequilibrium: the insulin gene region and insulin-dependent diabetes mellitus (IDDM). Am J Hum Genet 1993;52:506-16.

39 Abecasis GR, Cardon LR, Cookson WOC. A general test for association for quantitative traits in nuclear families. Am J Hum Genet 2000;66:279-92.

40 Nygaard TG, Wilhelmsen KC, Risch NJ, et al. Linkage mapping of doparesponsive dystonia (DRD) to chromosome 14q. Nat Genet 1993;5:386-91.

41 Suarez BK, Hampe CL. Linkage and association. Am J Hum Genet 1994; 54:554-9; discussion 560-3.

42 Risch N, Merikangas K. The future of genetic studies of complex human diseases. Science 1996;273:1516-7.

43 Lander E, Kruglyak L. Genetic dissection of complex traits: guidelines for interpreting and reporting linkage results. Nat Genet 1995;11:241-7.

44 Fasman KH, Letovsky SI, Li P, et al. The GDB Human Genome Database Anno 1997. Nucl Acids Res 1997:25:72-81.

45 Collins A, Frezal J, Teague J, et al. A metric map of humans: 23,500 loci in 850 bands. Proc Natl Acad Sci USA 1996;93:14771-5.

46 Collins A, Teague J, Keats BJ, et al. Linkage map integration. Genomics 1996;36:157-62. 